\title{
A phase I study of dasatinib with concurrent chemoradiation for stage III non-small cell lung cancer
}

\author{
Humera Khurshid, Thomas Dipetrillo, Thomas Ng, Kalyan Mantripragada, Ariel Birnbaum, David Berz, \\ Kathy Radie-Keane, Kimberly Perez, Maria Constantinou, Denise Luppe, Andrew Schumacher, \\ Kara Leonard and Howard Safran*
}

The Brown University Oncology Group, Providence, RI, USA

\section{Edited by:}

Minesh P. Mehta, Northwestern

University, USA

Reviewed by:

Daniel Grant Petereit, Rapid City

Regional Hospital, USA

Skye Hung-Chun Cheng, Koo

Foundation Sun Yat-Sen Cancer

Center, Taiwan

*Correspondence:

Howard Safran, Department of Medicine, Rhode Island Hospital, 593 Eddy Street, Providence, RI 02903 USA

e-mail: hsafran@lifespan.org

\begin{abstract}
Objectives: Src family kinases (SFKs) are expressed in non-small cell lung cancer (NSCLC) and may be involved in tumor growth and metastases. Inhibition of SFK may also enhance radiation. The purpose of this study was to evaluate if a maximum dose of $100 \mathrm{mg}$ of dasatinib could be safely administered with concurrent chemoradiation and then continued as maintenance for patients with newly diagnosed stage III NSCLC. Methods: Patients with stage III locally advanced NSCLC received paclitaxel, $50 \mathrm{mg} / \mathrm{m}^{2} /$ week, with carboplatin area under the curve $(A \cup C)=2$, weekly for 7 weeks, and concurrent radiotherapy, $64.8 \mathrm{~Gy}$. Three dose levels of dasatinib 50, 70, and $100 \mathrm{mg} /$ day were planned. Results: 11 patients with locally advanced NSCLC were entered. At the $70 \mathrm{mg}$ dose level 1 patient had grade 5 pneumonitis not responsive to therapy, and one patient had reversible grade 3 pneumonitis and grade 3 pericardial effusion. Due to these toxicities the Brown University Oncology Group Data Safety Monitoring Board terminated the study. Conclusion: Dasatinib could not be safely combined with concurrent chemoradiation for stage 3 lung cancer due to pneumonitis.
\end{abstract}

Keywords: lung cancer, radiation, Src, dasatinib

\section{INTRODUCTION}

Concurrent chemoradiation is the standard treatment for locally advanced stage III non-small cell lung cancer (NSCLC). However, the majority of patients will develop locoregional and distant metastases. RTOG 0617, a randomized phase III study of standard-dose ( $60 \mathrm{~Gy}$ ) versus high dose ( $74 \mathrm{~Gy})$ radiotherapy with concurrent and consolidation carboplatin/paclitaxel \pm cetuximab for patients with stage IIIA/IIIB NSCLC showed no survival benefit for high dose radiation (Bradley et al., 2011).

Additional chemotherapy either before or after chemoradiation also has not improved survival. The Hoosier Oncology Group (HOG) phase III trial demonstrated no improvement in outcome with the addition of three cycles of docetaxel $75 \mathrm{mg} / \mathrm{m}^{2}$ after definitive chemoradiotherapy as compared to chemoradiation alone (Hanna et al., 2008). The control group had a progression free survival of 12.9 months compared to 12 months for the docetaxel arm. Median overall survival was 24.1 months for the observation arm and 21.5 months for the docetaxel arm. CALGB 39801 demonstrated no improvement with induction chemotherapy prior to chemoradiation as compared to chemoradiation alone (Vokes et al., 2007). Two year overall survivals were 29 and 31\%, respectively. The addition of the EGFR small molecule tyrosine kinase inhibitor gefitinib after chemoradiotherapy and consolidation chemotherapy also failed to improve progression free survival or overall survival (Kelly et al., 2008).

An agent that could block the growth and prevent the development of micrometastases after locoregional therapy would have substantial importance. The Src family kinases (SFKs) are integral to many signaling pathways regulating cellular functions including proliferation, migration, and cellular survival (Kim et al., 2009). Activated SFKs are commonly expressed in NSCLC (Masaki et al., 2003; Zhang et al., 2007). Dasatinib is a potent inhibitor of SFKs (Haura et al., 2010; Johnson et al., 2010). Clinical studies of single agent dasatinib and the combination of dasatinib and erlotinib have demonstrated modest activity in NSCLC (Haura et al., 2010; Johnson et al., 2010). Inhibition of Src also enhances the sensitivity to radiation in NSCLC (Dittmann et al., 2009; Purnell et al., 2009).

The Brown University Oncology Group initiated a phase I study to determine whether dasatinib, up to a dose of $100 \mathrm{mg} /$ day could be safely administered with concurrent chemoradiation. The original protocol was designed to first evaluate dasatinib with chemoradiation for patients with unresectable NSCLC. If safety was established in this group of patients, the protocol planned for dasatinib to be investigated with neoadjuvant chemoradiation to $50.4 \mathrm{~Gy}$ followed by surgical resection in patients with potentially resectable stage IIIA disease. Since approximately one-third of patients with stage III NSCLC have grade $3 / 4$ non-hematologic toxicities with standard chemoradiation, the protocol allowed dose escalation to continue until three or more of six patients had dose limiting toxicities (DLT) at a given dose level. However, because of severe pneumonitis in two patients with inoperable lung cancer at the second dose level, this protocol was terminated by the Brown University Oncology Group Data Safety Monitoring Committee. This report describes protocol treatment and toxicity. 


\section{MATERIALS AND METHODS PATIENTS}

Patients with pathologically proven stage III NSCLC were eligible excluding patients with contralateral hilar lymphadenopathy. Patients with supraclavicular lymph node involvement were only eligible if they had an upper lobe primary tumor on the ipsilateral side of the supraclavicular lymph node. Patients could not have had prior chemotherapy or radiation for lung cancer. No prior radiation for any other malignancy within the radiation field was permissible. Patents with a pleural or pericardial effusion were excluded. Required diagnostic evaluation included history and physical examination, PET scan and CT or MRI of the brain. Mediastinoscopies were highly recommended. Patients must be at least 3 weeks from prior thoracotomy (if performed), ECOG performance status $0-1$, and age $>18$. Required laboratory parameters included absolute neutrophil count (ANC) $>1,500$ cells/ul, platelets $>100,000$ cells $/$ ul, hemoglobin $>9.0 \mathrm{~g} / \mathrm{dl}$, creatinine $<1.5 \times$ upper limit of normal (ULN), total bilirubin $<2.0 \times$ ULN, aspartate transaminase (AST), and alanine transaminase (ALT) $<2.5 \times$ the ULN, serum $\mathrm{Na}, \mathrm{K}^{+}, \mathrm{Mg}^{2+}$, phosphate, and $\mathrm{Ca}^{2+} \geq$ lower limit of normal (LLN). Patients could not have a severe active comorbidity.

\section{CHEMOTHERAPY}

Patients received paclitaxel, $50 \mathrm{mg} / \mathrm{m}^{2} /$ week and carboplatin, area under the curve $(\mathrm{AUC})=2$, weekly for 7 weeks with concurrent radiation. Prior to the first dosage of paclitaxel, patients were premedicated with dexamethasone $20 \mathrm{mg}$ orally the night before paclitaxel administration. Dexamethasone was administered either intravenously $30 \mathrm{~min}$ prior to paclitaxel administration or orally $1-3 \mathrm{~h}$ prior to paclitaxel administration. Prior to the first dosage of paclitaxel, patients were premedicated with diphenhydramine, $50 \mathrm{mg}$ intravenously, and ranitidine (or other $\mathrm{H} 2$ blocker), $50 \mathrm{mg}$ intravenously. If no allergic reactions occur, then subsequent dosages of dexamethasone, diphenhydramine, and $\mathrm{H} 2$ blockers could be reduced at the investigator's discretion.

\section{DASATINIB}

Dasatinib was administered orally on a daily basis throughout the 7 weeks of chemoradiation. Patients were treated with dasatinib according to a dose escalation scheme, with patients enrolled in cohorts of 3-6 at each dose level. Three dose levels 50, 70, and $100 \mathrm{mg} /$ day were planned. Beginning 28 days after completion of radiation, all patients would have dasatinib increased to $100 \mathrm{mg} /$ day and continued for 2 years or until disease progression or unacceptable toxicity.

\section{RADIOTHERAPY}

Radiation therapy was initiated with the first day of chemotherapy. 3D conformal treatment was delivered. The total dose to the involved areas for patients with unresectable NSCLC was 64.8 Gy in 35 fractions over 7 weeks. This was administered at $1.8 \mathrm{~Gy}$ daily, 5 days a week, for total of 25 fractions ( $45 \mathrm{~Gy}$ ) to the primary and mediastinum, followed by a boost to the primary and involved nodes to $1.8 \mathrm{~Gy}$ daily in 11 fractions (19.8 Gy). A volumetric treatment planning CT study was required to define gross tumor volumes (GTV), and planning target volume (PTV). Each patient was positioned in an individualized immobilization device in the treatment position on a flat table. Alpha cradle or approved alternate immobilization system was required.

Contiguous CT slices, 3-5 mm thickness of the regions harboring gross tumor and grossly enlarged nodes and $8-10 \mathrm{~mm}$ thickness of the remaining regions, were obtained starting from the level of the cricoid cartilage and extending inferiorly through the liver. The GTV, CTV, and PTV and normal organs were outlined on all appropriate CT slices and displayed using beam's eye view. A measurement scale for the CT image was included.

Normal tissues contoured included both lungs, skin, heart, spinal cord, esophagus, and liver. Dose constraints were defined such that the percent volume of bilateral lung receiving $5 \mathrm{~Gy}\left(\mathrm{~V}_{5 \mathrm{~Gy}}\right)$ was limited to $65 \%$ and the volume receiving $20 \mathrm{~Gy}\left(\mathrm{~V}_{20 \mathrm{~Gy}}\right)$ was limited to $35 \%$. The maximum dose to the heart was limited to $65 \mathrm{~Gy}$ or less; the volume of heart receiving $40 \mathrm{~Gy}$ was limited to $80 \%$ or less. The maximum dose to the esophagus was limited to $72 \mathrm{~Gy}$ or less and the mean dose to the esophagus was limited to $34 \mathrm{~Gy}$ or less.

\section{RESPONSE}

Response was evaluated using Response Evaluation Criteria in Solid Tumors (RECIST) criteria.

\section{STATISTICAL DESIGN}

The frequency of patients experiencing an acute DLT from the combination of dasatinib and chemoradiation was used for the determination of the maximum tolerated dose (MTD). The definition of DLT included acute grade 3 or grade 4 non-hematologic toxicity (excluding alopecia), grade 4 neutropenia or thrombocytopenia or any grade 5 toxicity related to treatment. For each arm, three to six patients were to be accrued. After it was determined that no more than three of six patients had DLTs when observed for at least 28 days after completion of radiation, the dose level was considered acceptable and the next dose level could be evaluated. Survival was calculated from the date of study entry.

The institutional review boards of all participating hospitals approved the study. All patients gave written informed consent according to federal and institutional guidelines.

\section{Table 1 | Patient characteristics.}

\section{Number of patients enrolled}

11

Median age in years (range)

$63(40-84)$

Male:Female

8:03

\section{CELLTYPE}

\section{Adenocarcinoma}

5

Squamous cell carcinoma

5

Non-small cell undetermined

1

\section{ECOG PERFORMANCE STATUS}

\section{0}

1

\section{STAGE}




\section{RESULTS}

\section{PATIENT CHARACTERISTICS}

Eleven patients with unresectable lung cancer were enrolled in this study between March 2009 and November 2010 and their characteristics are listed in Table 1. The median age was 63. Six patients had stage IIIA disease and five patients had stage IIIB disease. Nine patients had a performance status of 1 and two had a performance status of 0 .

\section{TOXICITY}

Six patients were entered at the dasatinib dose level of $50 \mathrm{mg} / \mathrm{day}$. Of these six patients, one withdrew consent and was not evaluable for toxicity. Of the remaining five patients, one had grade 3 esophagitis. Since four of five patients in this cohort successfully completed treatment, the protocol defined MTD of three or more of six patients developing DLT would not have been reached at the $50 \mathrm{mg}$ dose level. Therefore accrual to dose level 2, dasatinib $70 \mathrm{mg} /$ day with chemoradiation, was initiated.

Five patients were entered at dose level 2. Two of five patients developed pulmonary toxicity. One patient presented to the hospital 35 days after the last radiation dose with grade 3 pneumonitis and grade 3 pericardial effusion. This patient was immediately treated with prednisone with improvement of his symptoms and subsequent resolution. The $\mathrm{V}_{5 \mathrm{~Gy}}$ and $\mathrm{V}_{20 \mathrm{~Gy}}$ in this patient were 62 and $33 \%$, respectively and the volume of heart receiving 45 Gy was $15 \%$. These dose volume parameters are well within the constraints for lung and heart dose commonly used in cooperative group protocols. A second patient developed severe dyspnea 25 days after the last treatment with radiation. He had no prior history of lung disease, however his pretreatment DLCO was $52 \%$ of predicted prior to treatment. The $\mathrm{V}_{5 \mathrm{~Gy}}$ and $\mathrm{V}_{20 \mathrm{~Gy}}$ from his radiation treatment

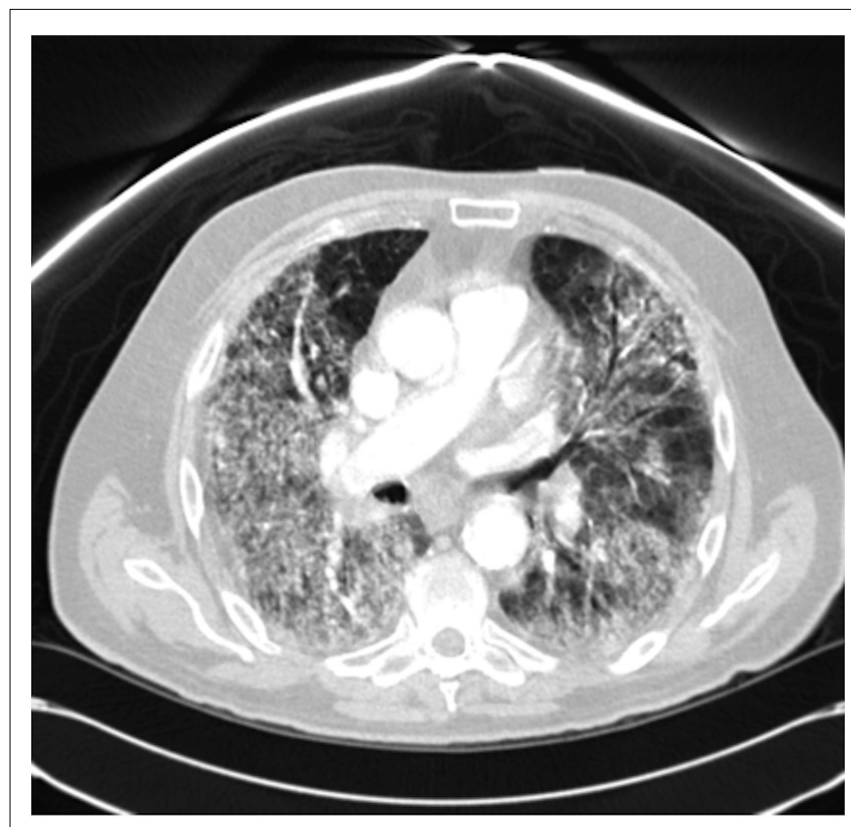

FIGURE 1 | Pneumonitis with pulmonary parenchymal abnormalities in a patient with stage IIIA NSCLC treated with dasatinib and concurrent chemoradiation. were 40 and $25 \%$, respectively. A CT scan of the chest done 10 days before the visit showed decrease in size of the lung mass from $3.1 \mathrm{~cm} \times 3.2 \mathrm{~cm}$ to $1.5 \mathrm{~cm} \times 1.8 \mathrm{~cm}$ and decrease in size of mediastinal lymph node and a faint diffuse reticulonodular pattern in the lungs. Repeat CT chest on admission with showed extensive bilateral predominantly basilar diffuse interstitial disease as shown in Figure 1 below.

Bronchoscopy was performed. All bacteriologic, viral, and fungal cultures were negative and respiratory viral panel was negative. He was treated with broad spectrum antibiotics, corticosteroids, and diuretics. An echocardiogram showed right ventricular systolic pressure (RVSP) of $71 \mathrm{~mm} \mathrm{Hg}$, indicating moderate pulmonary hypertension. The patient's respiratory status continued to worsen and the patient expired. Acute toxicities of all 10 patients assessable for toxicity are listed in Table 2. Multiple toxicities in a single patient are scored as separate events.

Table 2 | Toxicity*.

Number of patients $(n=10)$

Grade 2 Grade $3 \quad$ Grade 4

NON-HEMATOLOGICAL

1. Gastrointestinal

Nausea

Vomiting

Diarrhea

Dehydration

Heartburn

Esophagitis

Dysphagia

2. Constitutional

Fatigue

Anorexia

Weight loss

3. Cardiac

Chest pain

Troponin elevation

Cardiac ischemia

Pericardial effusion

Hypotension

Arrhythmia

4. Pulmonary

Pneumonitis

Dyspnea

Pleural effusion

5. Infections

Infection with normal ANC

6. Allergies

Carboplatin

HEMATOLOGICAL

Neutropenia

Anemia

Thrombocytopenia

\section{Grade 2}

Grade 3

\section{Grade 4}

Thrombocytopenia

*Multiple toxicities in the same patient are scored as separate events. 
Of the 10 assessable patients accrued, two had a partial response. The median survival is 18 months. Five patients remain alive at 18, 21, 27, 27, and 32 month follow-up.

\section{DISCUSSION}

The Brown University Oncology Group sought to investigate the addition of a Src inhibitor to chemoradiation for stage 3 NSCLC. Exposure to ionizing radiation can activate the epidermal growth factor receptor (Schmidt-Ullrich et al., 1997). Src kinase activation plays a crucial role during this process. Radiation activated Src kinase initiates EGFR internalization and nuclear transport (Khan et al., 2006). Purnell et al. (2009) reported that Src was activated in four of five lung cancer cell lines and the level corresponded with the invasive potential. Submicromolar concentrations of the Src inhibitor AZD0530 blocked Src and focal adhesion kinase, resulting in significant inhibition of cell migration and invasion. Src inhibition gave a potent and sustained blockage of AKT and enhanced sensitivity to radiation (Purnell et al., 2009).

Dasatinib has been evaluated in clinical studies in NSCLC. Johnson et al. (2010) evaluated single agent dasatinib in patients with advanced non-small-cell lung cancer. Thirty-four patients were enrolled. The first 22 patients received dasatinib $100 \mathrm{mg}$ twice a day, however, because of poor tolerance (fatigue and pleural effusion), the remaining 11 patients received dasatinib $100 \mathrm{mg}$ in the morning and $50 \mathrm{mg}$ in the evening. Grade 3 dyspnea occurred in 15 patients (44\%); 6 (18\%) developed grade 3 pleural effusion. Pleural effusions generally resolved with a short course of steroids, diuretics, and temporary interruption with dasatinib. The overall disease control rate (partial responses plus stable disease) for dasatinib was $43 \%$. One patient had a partial response to therapy and 11 patients $(32 \%)$ had a metabolic response by PET scan. The presence of a pleural effusion before dasatinib therapy predicted the development of a clinically significant effusion during therapy.

Haura et al. (2010) performed a phase I/II study of dasatinib with erlotinib in 30 patients with NSCLC. The average duration of treatment was 73 days. Two partial responses and one bone response were observed and the disease control rate was $63 \%$. Six patients (18\%) had grade 2 pleural effusions. Other toxicities

\section{REFERENCES}

Bergeron, A., Réa, D., Levy, V., Picard, C., Meignin, V., Tamburini, J., Bruzzoni-Giovanelli, H., Calvo, F., Tazi, A., and Rousselot, P. (2007). Lung abnormalities after dasatinib treatment for chronic myeloid leukemia: a case series. Am. J. Respir. Crit. Care Med. 176, 814-818.

Bradley, J. D., Paulus, R., Komaki, R., Masters, G., Forster, K., Schild, S. E., Bogart, J. A., Garces, Y. I., Narayan, S., and Choy, H. (2011). "Randomized phase III comparison of standarddose (60 Gy) versus high-dose (74 GY) conformal chemoradiotherapy with or without cetuximab for stage IIIA/IIIB non-small cell lung cancer: preliminary findings on radiation dose in RTOG 0617," in 53rd ASTRO Annual Meeting, Miami Beach.

Dittmann, K., Mayer, C., Kehlbach, R., Rothmund, M. C., and Peter Rodemann, H. (2009). Radiationinduced lipid peroxidation activates src kinase and triggers nuclear EGFR transport. Radiother. Oncol. 92, 379-382.

Dumitrescu, D., Seck, C., ten Freyhaus, H., Gerhardt, F., Erdmann, E., and Rosenkranz, S. (2011). Fully reversible pulmonary arterial hypertension associated with dasatinib treatment for chronic myeloid leukaemia. Eur. Respir. J. 38, 218-220.

Hanna, N., Neubauer, M., Yiannoutsos, C., McGarry, R., Arseneau, J., Ansari, R., Reynolds, C., Govindan, R., Melnyk, A., Fisher,

included diarrhea, anorexia, nausea, rash, hematologic toxicity, and fatigue (Haura et al., 2010).

Concurrent chemoradiation can cause pulmonary toxicity. In CALGB 39801 the incidence of grade 3 and grade 4 pneumonitis in patients receiving chemoradiation (without induction chemotherapy) was 3 and 1\%, respectively (Vokes et al., 2007). The Brown University sought to establish whether dasatinib could be safely added to chemoradiation. To minimize patient risk, patients with any pleural effusion were not eligible for this study. A maximum dose of $100 \mathrm{mg}$ /day was targeted based on a randomized phase III study in patients with Philadelphia chromosome positive hematologic malignancies (Shah et al., 2008). Compared with the $70 \mathrm{mg}$ twice-daily regimen, dasatinib $100 \mathrm{mg}$ once daily resulted in significantly lower rates of pleural effusion (all grades 7 versus $16 \% ; p=0.024$ ) and grade $3-4$ thrombocytopenia (22 versus $37 \%, p=0.004)$ and fewer patients required dose interruption, reduction, or discontinuation (Shah et al., 2008).

However, the $100 \mathrm{mg}$ target dose could not be achieved in this phase I study. Fatal pneumonitis, complicated by pulmonary hypertension in one patient, and a second patient with reversible pneumonitis and pericardial effusion, developed at the $70 \mathrm{mg}$ dose level. In the patient who developed grade 5 toxicity, in addition to pleural effusion, the patient developed parenchymal pulmonary abnormalities and pulmonary hypertension. There are four separate case reports of pulmonary hypertension with dasatinib (Mattei et al., 2009; Rasheed et al., 2009; Dumitrescu et al., 2011; Orlandi et al., 2012). Parenchymal changes (ground glass or alveolar opacities and septal thickening) with and without pleural effusion has also been described (Bergeron et al., 2007). It is possible that the pneumonitis observed in this study was due to chemoradiation alone or that a safe low dose of dasatinib could possibly have been detected. However, due to the severity of the toxicity, combined with other case reports of pulmonary hypertension and its irreversible nature, the Brown University Oncology Group Data Safety Monitoring Committee recommended study closure.

\section{ACKNOWLEDGMENTS}

Disclosures: Supported in part by Bristol Myers Squibb.

W., Richards, D., Bruetman, D., Anderson, T., Chowhan, N., Nattam, S., Mantravadi, P., Johnson, C., Breen, T., White, A., and Einhorn, L. (2008). Phase III study of cisplatin, etoposide and concurrent chest radiation with or without consolidation docetaxel in patients with inoperable stage III non-small cell lung cancer: the Hoosier Oncology Group and U.S. Oncology. J. Clin. Oncol. 26, 5755-5760.

Haura, E. B., Tanvetyanon, T., Chiappori, A., Williams, C., Simon, G., Antonia, S., Gray, J., Litschauer, S., Tetteh, L., Neuger, A., Song, L., Rawal, B., Schell, M. J., and Bepler, G. (2010). Phase I/II study of the src inhibitor dasatinib in combination with erlotinib in advanced non-small-cell lung cancer. J. Clin. Oncol. 28, 1387-1394.

Johnson, F. M., Bekele, B. N., Feng, L., Wistuba, I., Tang, X. M., Tran, H. T., Erasmus, J. J., Hwang, L. L., Takebe, N., Blumenschein, G. R., Lippman, S. M., and Stewart, D. J. (2010). Phase II study of dasatinib in patients with advanced non-smallcell lung cancer. J. Clin. Oncol. 28, 4609-4615.

Kelly, K., Chansky, K., Gaspar, L. E., Albain, K. S., Jett, J., Ung, Y. C., Lau, D. H., Crowley, J. J., and Gandara, D. R. (2008). Phase III trial of maintenance gefitinib or placebo after concurrent chemoradiotherapy and docetaxel consolidation in inoperable stage III non-small cell lung cancer. J. Clin. Oncol. 26, 2450-2456. 
Khan, E. M., Heidinger, J. M., Levy, M., Lisanti, M. P., Ravid, T., and Goldkorn, T. (2006). Epidermal growth factor receptor exposed to oxidative stress undergoes Srcand caveolin-1-dependent perinuclear trafficking. J. Biol. Chem. 281, 14486-14493.

Kim, L. C., Song, L., and Haura, E. B. (2009). Src kinases as therapeutic targets for cancer. Nat. Rev. Clin. Oncol. 6, 587-595.

Masaki, T., Igarashi, K., Tokuda, M., Yukimasa, S., Han, F., Jin, Y. J., Li, J. Q., Yoneyama, H., Uchida, N., Fujita, J., Yoshiji, H., Watanabe, S., Kurokohchi, K., and Kuriyama, S. (2003). pp60c- src activation in lung adenocarcinoma. Eur. J. Cancer 39, 1447-1455.

Mattei, D., Feola, M., Orzan, F., Mordini, N., Rapezzi, D., and Gallamini, A. (2009). Reversible dasatinibinduced pulmonary arterial hypertension and right ventricle failure in a previously allografted CML patient. Bone Marrow Transplant. 43, 967-968.

Orlandi, E. M., Rocca, B., Pazzano, A. S., and Ghio, S. (2012). Reversible pulmonary arterial hypertension likely related to long-term, lowdose dasatinib treatment for chronic myeloid leukemia. Leuk. Res. 36, e4-e6.

Purnell, P. R., Mack, P. C., Tepper, C. G., Evans, C. P., Green, T. P., Gumerlock, P. H., Lara, P. N., Gandara, D. R., Kung, H. J., and Gautschi, O. (2009). The Src inhibitor AZD0530 blocks invasion and may act as a radiosensitizer in lung cancer cells. J. Thorac. Oncol. 4, 448-454.

Rasheed, W., Flaim, B., and Seymour, J. F. (2009). Reversible severe pulmonary hypertension secondary to dasatinib in a patient with chronic myeloid leukemia. Leuk. Res. 33, 861-864.

Schmidt-Ullrich, R. K., Mikkelsen, R. B., Dent, P., Todd, D. G., Valerie, K., Kavanagh, B. D., Contessa, J. N., Rorrer, W. K., and Chen, P. B. (1997). Radiation-induced proliferation of the human A431 squamous carcinoma cells is dependent on EGFR tyrosine phosphorylation. Oncogene 15, 1191-1197.

Shah, N. P., Kantarjian, H. M., Kim, D. W., Réa, D., Dorlhiac-Llacer, P. E., Milone, J. H., Vela-Ojeda, J., Silver, R. T., Khoury, H. J., Charbonnier, A., Khoroshko, N., Paquette,
R. L., Deininger, M., Collins, R. H., Otero, I., Hughes, T., Bleickardt, E., Strauss, L., Francis, S., and Hochhaus, A. (2008). Intermittent target inhibition with dasatinib 100 mg once daily preserves efficacy and improves tolerability in imatinibresistant and intolerant chronicphase chronic myeloid leukemia. J. Clin. Oncol. 26, 3204-3212.

Vokes, E. E., Herndon, J. E. II, Kelley, M. J., Cicchetti, M. G., Ramnath, N., Neill, H., Atkins, J. N., Watson, D. M., Akerley, W., and Green, M. R. (2007). Induction chemotherapy followed by chemoradiotherapy compared with chemoradiotherapy alone for regionally advanced unresectable stage III non-small-cell lung cancer: cancer and leukemia group B. J. Clin. Oncol. 25, 1698-1704.

Zhang, J., Kalyankrishna, S., Wislez, M. Thilaganathan, N., Saigal, B., Wei, W., Ma, L., Wistuba, I. I., Johnson, F. M., and Kurie, J. M. (2007). SRC-family kinases are activated in non-small cell lung cancer and promote the survival of epidermal growth factor receptor-dependent cell lines. Am. J. Pathol. 170, 366-376.
Conflict of Interest Statement: The authors declare that the research was conducted in the absence of any commercial or financial relationships that could be construed as a potential conflict of interest.

Received: 22 April 2012; accepted: 13 May 2012; published online: 31 May 2012.

Citation: Khurshid H, Dipetrillo $\mathrm{T}, \mathrm{Ng}$

T, Mantripragada K, Birnbaum A, Berz $D$, Radie-Keane K, Perez K, Constantinou $M$, Luppe D, Schumacher A, Leonard K and Safran H (2012) A phase I study of dasatinib with concurrent chemoradiation for stage III non-small cell lung cancer. Front. Oncol. 2:56. doi: 10.3389/fonc.2012.00056

This article was submitted to Frontiers in Radiation Oncology, a specialty of Frontiers in Oncology.

Copyright (C) 2012 Khurshid, Dipetrillo, $\mathrm{Ng}$, Mantripragada, Birnbaum, Berz, Radie-Keane, Perez, Constantinou, Luppe, Schumacher, Leonard and Safran. This is an open-access article distributed under the terms of the Creative Commons Attribution Non Commercial License, which permits non-commercial use, distribution, and reproduction in other forums, provided the original authors and source are credited. 